\title{
Agentes causantes de Bacteriemia en pacientes adultos con Neutropenia febril del Hospital de Clínicas. Paraguay ${ }^{4}$ Bacteremia-causing agents in adult patients with febrile neutropenia at the Hospital de Clínicas. Paraguay
}

\author{
Víctor Daniel Giménez Ortigoza ${ }^{5}$
}

María Cristina San Miguel $^{6}$

\section{DOI: https://doi.org/10.36003/Rev.investig.cient.tecnol.V4N1(2019)2}

\section{RESUMEN}

La neutropenia febril es una de las complicaciones más frecuentes y de mayor morbilidad y mortalidad en los pacientes hematológicos. La cambiante tendencia en etiología de la bacteriemia y el patrón de susceptibilidad antimicrobiana comprometen la tasa de respuesta a los esquemas de tratamiento empírico. El objetivo fue determinar la frecuencia de bacterias aisladas en pacientes con neutropenia febril del Hospital de Clínicas. Se revisaron las historias clínicas de los pacientes con diagnóstico de neutropenia febril internados en la 3 CCM en el HC-FCM desde enero del año 2016 a enero del año 2019. La información se analizó utilizando Excell y Epiinfo. Se revisaron un total de 95 fichas clínicas de las cuales 72 cumplieron con criterios para ser incluidas en el presente estudio, la edad promedio de los pacientes fue de 38,63 $\pm 17,8$ años, una frecuencia de 40 $\%$ de bacteriemia, predominio etiológico de Cocos gram positivos, un promedio de 19,7 días de estancia hospitalaria. Las especies microbianas aisladas con mayor frecuencia (klebsiella pneumoniae y Staphylococcus aureus).

Palabras clave: Neutropenia; bacteriemia; hematología; inmunosupresión.

\footnotetext{
${ }^{4}$ Fecha de recepción: octubre 2019; fecha de aceptación: noviembre 2019

${ }^{5}$ Especialista en Medicina Interna. Hospital de Clínicas - Facultad de Ciencias Médicas de la Universidad Nacional de Asunción. San Lorenzo, Paraguay

${ }^{6}$ Especialista en Infectología del Instituto de Medicina Tropical, Asunción, Paraguay
} 


\section{ABSTRACT}

Febrile neutropenia is one of the most frequent complications with the highest morbidity and mortality in haematological patients. The changing trend in the etiology of bacteremia and the pattern of antimicrobial susceptibility compromise the response rate to empirical treatment regimens. The objective was to determine the frequency of isolated bacteria in patients with febrile neutropenia at the Hospital de Clínicas. The medical records of patients diagnosed with febrile neutropenia hospitalized in the 3 CCM in the HC-FCM from January 2016 to January 2019 were reviewed. The information was analyzed using Excell and Epiinfo. A total of 95 clinical records were reviewed, of which 72 met the criteria to be included in the present study. The average age of the patients was $38.63 \pm 17.8$ years, a $40 \%$ frequency of bacteremia, predominantly aetiological Gram-positive cocci, an average of 19.7 days of hospital stay. The most frequently isolated microbial species (klebsiella pneumoniae and Staphylococcus aureus).

Keywords: Neutropenia; bacteremia; hematology; immunosuppression. 


\section{INTRODUCCIÓN}

La neutropenia febril (NF) es una de las complicaciones más frecuentes y de mayor morbimortalidad entre los pacientes que padecen una neoplasia. La neutropenia si bien ocurre con mayor frecuencia en patologías oncológicas no es específica de pacientes con cáncer y puede observarse en otras enfermedades en las que exista una noxa que favorezca la granulocitopenia. Durante estos episodios de neutropenia la fiebre es una complicación usual, con una frecuencia de 6 a $8 \%$ después de la quimioterapia convencional y hasta $40 \%$ en pacientes que reciben quimioterapia intensiva (1-3).

En un paciente con neutropenia se considera fiebre a la temperatura mayor de 38.3 ${ }^{\circ} \mathrm{C}$ en una ocasión o mayor de $38^{\circ} \mathrm{C}$ por más de una hora. La neutropenia grave se define como la cuenta absoluta de neutrófilos $<500$ células/ microL y la neutropenia prolongada cuando dura más de siete días $(4,5)$.

En los pacientes con NF se documentan infecciones por bacterias, hongos y menos frecuentemente por virus. Las bacterias son los agentes etiológicos más frecuentemente involucrados en las fases iniciales aislándose tanto bacilos gramnegativos (BGN) como cocos grampositivos (CGP). En cambio, las infecciones por hongos son infrecuentes como infección primaria, salvo Candida sp, que ocasionalmente puede ser identificada en hemocultivos $(6,7)$.

La infección en el paciente neutropénico constituye un desafío permanente. Si bien las estrategias de manejo han mejorado en los últimos años, el aumento de la prevalencia de pacientes oncológicos, los avances alcanzados por la modificación de la intensidad de los esquemas de tratamiento, la aparición de nuevos citostáticos, las variaciones observadas en la etiología de las infecciones y la emergencia de resistencia antimicrobiana son circunstancias problemáticas constantes, debe ser entendida como una emergencia médica por lo que es primordial reconocerla $(7,8,9)$.

Si bien se sabe que los eventos de NF están determinados por distintas variables, que su frecuencia, presentación clínica, hallazgos microbiológicos y evolución dependen de diferentes factores, es importante y necesario conocer las características propias de esta entidad a nivel local, contextualizándola en el marco regional. La experiencia recogida por la atención de este síndrome pone en evidencia la necesidad de adaptarla a nuestra realidad socioeconómica, con el empleo racional de los recursos, en función de una relación costo-beneficio favorable y reducción de la mortalidad.

\section{Objetivo General:}

Determinar la frecuencia de bacterias aisladas en pacientes con neutropenia febril en la III CCM del Hospital de Clínicas. San Lorenzo-Paraguay en el período de 2016 al 2019.

Objetivos Específicos:

1. Características demográficas de los pacientes con neutropenia febril.

2. Determinar las causas de Neutropenia febril más frecuentes en el Hospital de Clínicas.

3. Determinar mortalidad en pacientes con neutropenia febril.

4. Conocer la frecuencia de utilización de vías de acceso vascular central en pacientes con neutropenia febril.

5. Conocer la frecuencia de los antibióticos utilizados en pacientes con neutropenia febril.

6. Conocer la estancia hospitalaria de pacientes con neutropenia febril. 


\section{MÉTODO}

Estudio observacional descriptivo de corte transverso con componentes analíticos. Muestreo: no probabilístico de casos consecutivos de los pacientes con neutropenia febril internados en la III catedra de Clínica médica del Hospital de Clínicas - Facultad de Ciencias Médicas de la Universidad Nacional de Asunción. (San Lorenzo, Paraguay). Espacio geográfico: III Cátedra de Clínica Médica del Hospital de Clínicas -FCM. Determinación temporal: Período de enero del año 2016 a enero del año 2019. Población de enfocada: pacientes con diagnóstico de neutropenia febril. Población de estudio: pacientes internados con diagnóstico de neutropenia febril en la III CCM del Hospital de Clínicas. San Lorenzo-Paraguay en el período de 2016 al 2019.

\section{Criterios de inclusión:}

Pacientes con temperatura mayor a $38^{\circ} \mathrm{C}$ en una ocasión durante la internación y neutropenia grave (recuento de neutrófilos menor o igual 500 células/micro).

Pacientes con diagnóstico confirmado de neutropenia febril internados en la III Cátedra de Clínica Médica del Hospital de Clínicas durante el periodo de estudio.

Pacientes Mayores de 18 años.

Pacientes que tuvieron hemocultivos positivos durante fase neutropénica

\section{Criterios de Exclusión:}

Pacientes con temperatura mayor a $38^{\circ} \mathrm{C}$ en una ocasión durante la internación y neutropenia grave, pero con diagnóstico de ingreso de VIH/SIDA.

Paciente con Historias clínicas incompletas o dañadas.
Pacientes que no se han realizados los hemocultivos durante la fase neutropénica o que el diagnostico confirmatorio no haya sido bacteriano.

\section{Variables de estudio:}

Cuantitativa: edad, días de internación.

Cualitativa: sexo, bacteriemia, causas de neutropenia febril, antibioticoterapia, presencia de vía venosa central y óbito

\section{Recolección de datos}

Se completaron fichas de datos a partir de las historias clínicas de pacientes que ingresaron con diagnóstico de neutropenia y que refirieron hipertermia o desarrollaron hipertermia durante la internación mayor a $38^{\circ} \mathrm{C}$ y ocasiono la realización del hemocultivo para descartar infecciones bacterianas. De hallar el mismo positivo se registró el agente etiológico.

\section{Cálculo del tamaño Muestral:}

Para una frecuencia estimada de cultivos positivos de $48 \%$ en pacientes con Neutropenia febril (10) con un nivel de confianza del $95 \%$, el tamaño de la muestra calculada fue de 72 participantes.

\section{Gestión y Análisis de Datos.}

Las variables se registraron en planillas electrónicas Excel. Las cualitativas de expresaran en frecuencias y porcentajes, las cuantitativas en medias y desvío estándar. Para las asociaciones se utilizó el programa Epi info y los estadísticos utilizados fueron OR, T student y kruskal-Wallis.

\section{Consideraciones Éticas:}

Fue respetada la confidencialidad y los principios básicos de la Ética de los pacientes. Para la utilización de las fichas clínicas se solicitó autorización del jefe del servicio.

\section{RESULTADOS}

Se revisaron un total de 95 fichas clínicas de las cuales $72(75 \%)$ cumplieron con criterios para ser incluidas en el presente estudio, la edad promedio de los pacientes fue de $38,63 \pm 17,8$ años (Gráfico 1) siendo el $52 \%$ de sexo femenino y el $48 \%$ de sexo masculino. 


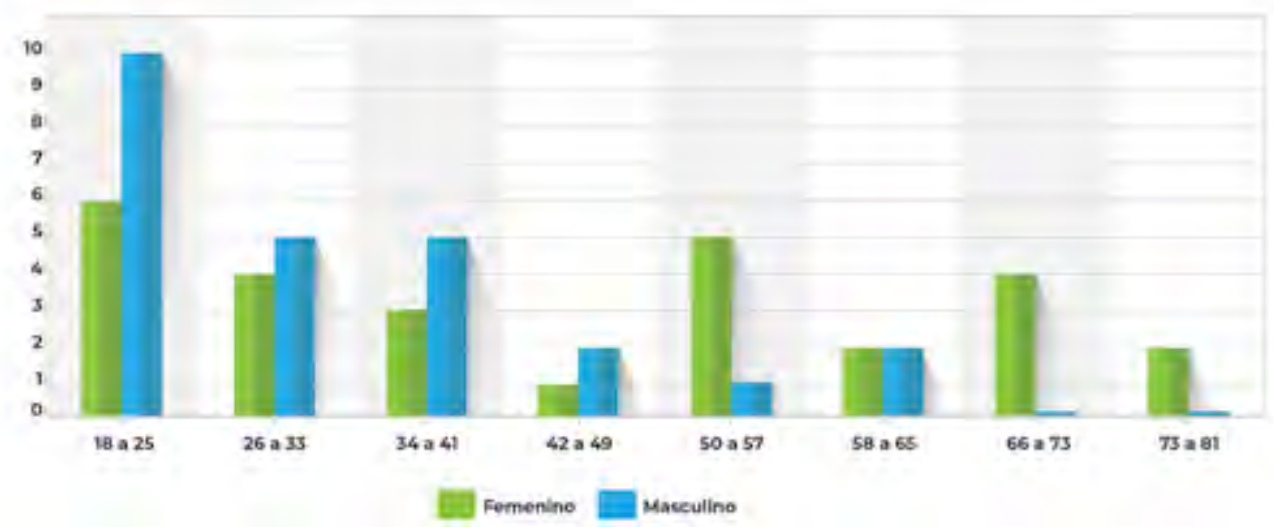

Gráfico 1. Distribución de pacientes con Neutropenia Febril internados en la IIICCM del Hospital de Clínicas por grupo etario y Sexo. $n=52$

En cuanto a la causa de neutropenia en $19(31 \%)$ de los casos fueron secundarios a una LLA, 11(15\%) secundarios a una
LMA, $10(14 \%)$ de los casos fue por Quimioterapia y $5(6 \%)$ de los casos secundarios a un linfoma no hodking (Gráfico 2).

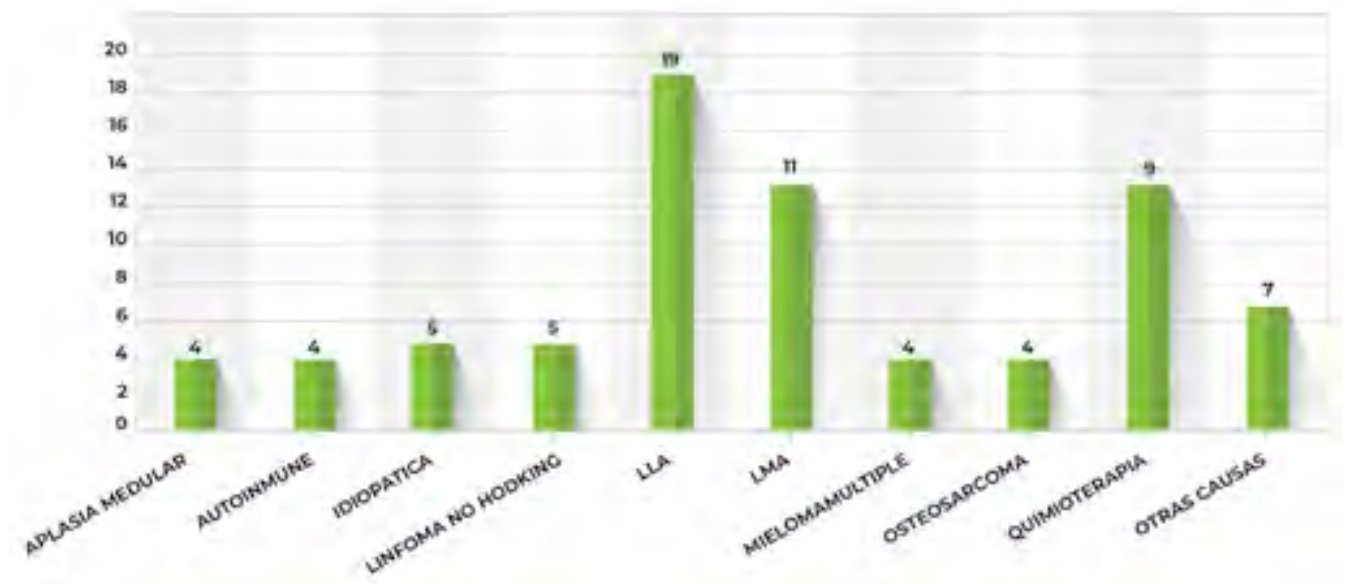

Gráfico 2. Causas de Neutropenia febril en pacientes de la III CCM del Hospital de Clínicas. n=72

Los pacientes permanecieron internados en promedio $19,77 \pm 15,06$ días en los cuales se le tomaron en promedio $1,9 \pm$ 1,52 pares de hemocultivos. En general los pacientes en un 44\% ( n:31) de los casos fueron tratados con múltiples antibióticos, siendo el más frecuentemente utilizado el tazonam en 37 (51\%) pacientes, seguido por la vancomicina en $18(25 \%)$ pacientes y ceftriaxona en $6(5 \%)$ pacientes (Grafico 3 ). 


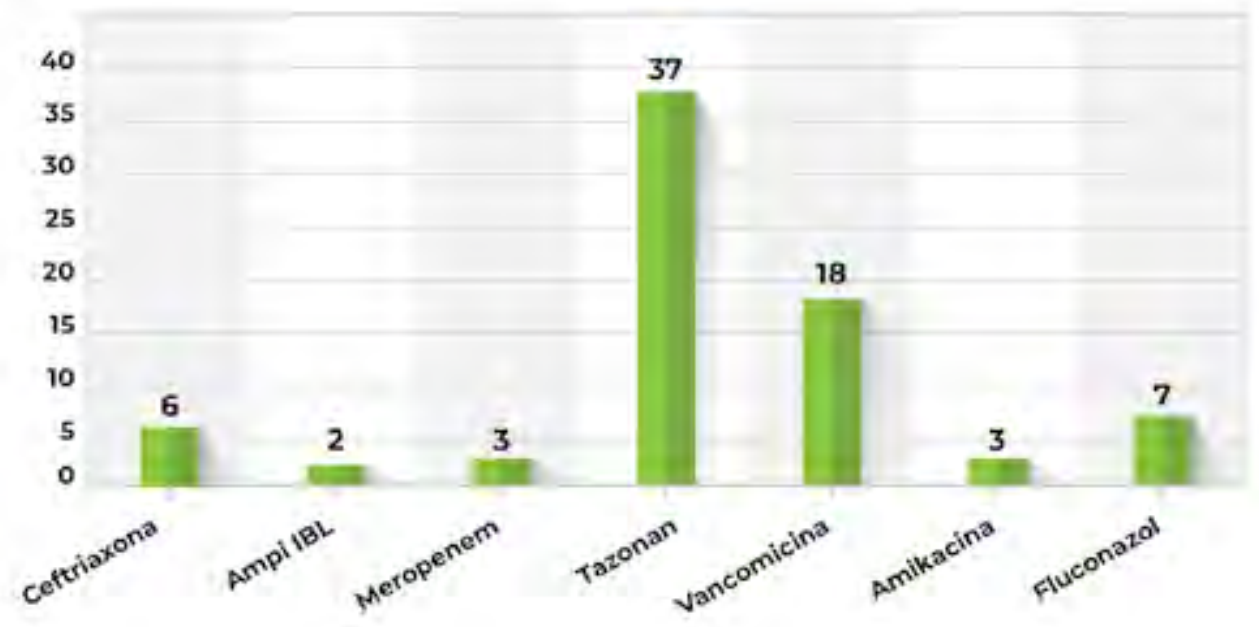

Gráfico 3. Antibióticos utilizados en pacientes con neutropania febril de la III CCM en el Hospital de Clínicas. $\mathrm{n}=52$

Solo el 32\% (n:44) presento una vía venosa central, en un $26 \%$ ( n:19) de los pacientes fue aislado un germen y en de los cuales en un $28 \%$ (n:20) de los pacientes fue aislado más de un germen. En cuanto a los gérmenes aislados el más frecuente fue la Klebsiella pneumoniae en 6 oportunidades ( $28 \%$ ) de los cuales en
1 oportunidad fue carbapenem resistente y en 2 ocasiones tipo BLEE, seguido por Staphylococcus aureus en 4 pacientes ( $19 \%$ ) de los cuales 2 eran meticilino resistente y el Staphycoccus epidermidis en 3 pacientes(14\%) ( Grafico 4 ).

Solo se produjo el óbito de 4 pacientes $(5 \%)$.

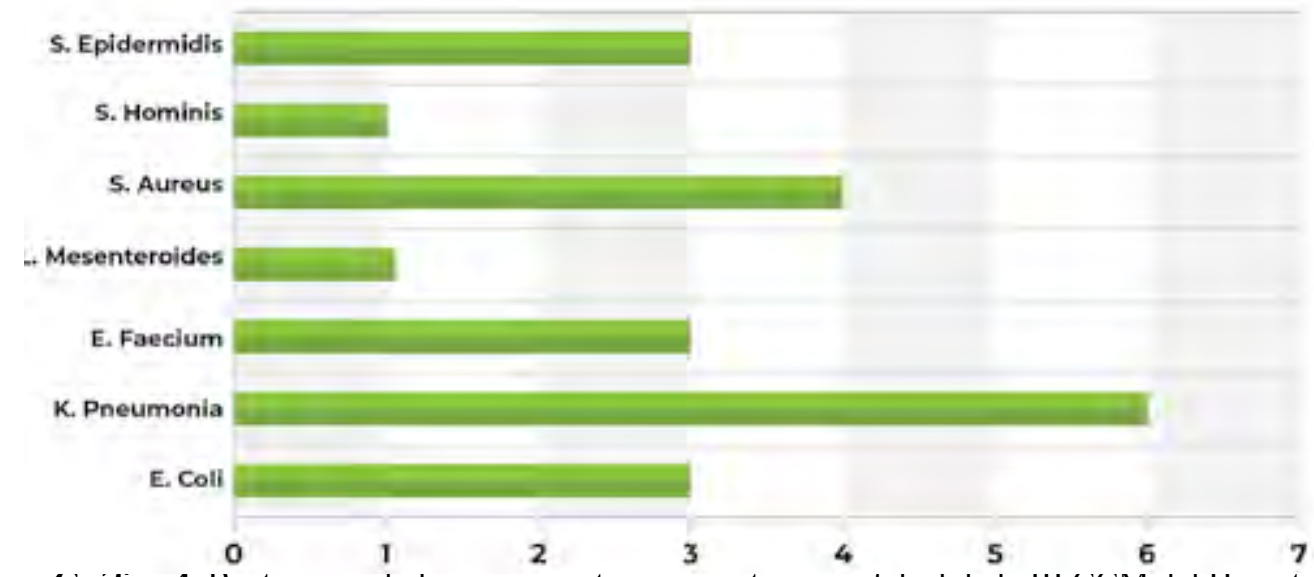

Gratico 4. Bacterias aisladas en pacientes con neutropenia tebril de la III LCM del Hospital de Clínicas. N: 21

No se hallaron asociaciones significativas entre la presencia de una vía central y el óbito de los pacientes (OR 4,53 IC 0,38 - 53, 93 p: 0,24), entre la presencia de un germen aislado y el óbito de los pacientes
(OR 6,17 IC 0,51 - 74,17 p: 0,17), entre la presencia de vía central y el aislamiento de gérmenes (OR 2,8 IC 0,78 - 9,99 p:0,18).

Se hallaron diferencias significativas entre la media de días de internación entre los 
pacientes y la presencia de múltiples gérmenes aislado (p: 0,00001), entre los días de internación y la presencia de germen aislado (p 0,0003)

\section{DISCUSIÓN}

En este estudio el promedio de edad fue de 38 años y la distribución de sujetos según edad llama la atención donde se ve que la mayor parte de casos masculinos de neutropenia febril son jóvenes a diferencia de las mujeres que predominan en edades avanzadas.

El $30 \%$ de los pacientes con NF fueron secundarias leucemias agudas superior a estudios similares en Colombia (11) y México (10) donde las leucemias correspondían al $19 \%$ de la población, este dato puede deberse debido a la gran cantidad de pacientes hematológicos en nuestro servicio por la falta de otro centro público en el país donde se tratarían este tipo de pacientes.

La frecuencia de episodios de NF con bacteriemia confirmada fue de $30 \%$, superior a lo referido en la literatura científica de países desarrollados $(12,13)$ pero similar a otros estudios latinoamericanos $(10,14)$. El porcentaje de óbito en el estudio fue menor a los registrados en el México (10), Argentina (15) donde llegan al $10 \%$.

Los antibióticos utilizados más frecuentemente fueron Tazonan $51 \%$ y Vancomicina $25 \%$ que según las guías IDSA (13) y regionales $(15,11)$ son primera línea en el tratamiento empírico de neutropenia febril, llama la atención el aislamiento del género Leuconostop mesenteroides que son intrínsecamente resistentes a glucopeptidos (16).

Existen pocos datos sobre la etiología bacteriana en neutropenia febril a nivel Mercosur. Se observa un predomino las bacterias gran positivas $57 \%$ (n:13) a diferencia de estudios similares en Perú (17), México (10) y Chile (18) donde predominó los grandes negativos, las causas de estas diferencias podrían ser muchas entre ellas epidemiologia local, predominancia de infecciones de piel, mal manejo de vías venosas, etc.

Las especies microbianas aisladas con mayor frecuencia (Klebsiella pneumoniae y Sthaphylococcus aureus), fueron similares a las encontradas en otras series de Perú (17) y Colombia (11). Cabe resaltar que en nuestro centro a pesar de recomendación internacionales $(13,16)$ no se produce el aislamiento de los pacientes neutrópenicos por falta de disponibilidad de salas especiales y recursos hospitalarios.

El 32\% de los pacientes presento una vía venosa central, una frecuencia menor a países desarrollados (13), No se encontró asociación significativa entre la presencia de vía venosa central y la presencia de bacteriemia y el óbito; a pesar que inicialmente pensamos encontrar lo contrario debido a la posible puerta de entrada que implicaría la misma.

La estancia prolongada es una realidad en nuestro medio en este tipo de paciente con un promedio de 19,7 días, evidentemente eso predispone a complicaciones diversas entre ellas las infecciosas, se encontró asociación significativa entre los días de internación y la presencia de germen aislado (p 0,003).

Este estudio tiene limitaciones, la posibilidad de datos incompletos o no reportados en la historia clínica, no incluimos diagnósticos infecciosos y no están los datos de exposición previa a antimicrobianos como profilaxis; la falta de toma de hemocultivos en ciertos en pacientes neutropénicos por no disponibilidad de recursos económicos para solventarlos. Si bien este estudio se refiere sólo a la experiencia de un centro, demuestra la importancia de la 
vigilancia de los episodios de NF para definir conductas locales; la generalización de estos datos no es posible, pero pueden ser orientadores para centros que atiendan pacientes adultos con patologías similares.

El presente estudio es relevante porque aporta datos acerca de la situación actual de los pacientes con neutropenia febril internados en el hospital de clínicas además de los agentes etiológicos para poder ajustar la terapia empírica; la abundancia de paciente con neoplasia hematológicas, neutropénicos por quimioterapia, agranulocitosis, etc. nos obliga a tomar medidas y seguir investigando como centro hospitalario los posibles factores de riesgo, mejorar el manejo para lograr disminuir la morbimortalidad y la estancia prolongada en estos pacientes.

\section{CONCLUSIONES}

La frecuencia de bacteriemia en pacientes con NF es alta; similar a países de la región, pero mayor a países de primer mundo.

La mayoría de los pacientes jóvenes con NF son del sexo masculino y en contrapartida los adultos fueron femeninos.

Las causas más frecuentes de neutropenia fueron las leucemias, hallazgo esperado por la gran cantidad de pacientes hematológicos que acuden a nuestro hospital.

La mortalidad fue menor en compara- ción a estudios de la región.

La frecuencia de utilización de accesos venosos vasculares es baja pero similar a países de la región.

El antibiótico más utilizado en el estudio fue para gérmenes gran negativos, coincidiendo con las recomendaciones de guías internaciones.

La estancia prolongada es una realidad en nuestro servicio y se halló una asociación significativa con el Óbito de los pacientes en el estudio.

\section{BIBLIOGRAFÍA}

1. Ammann RA, Niggli FK, Leibundgut K, Teuffel O, Bodmer N. Exploring the association of hemoglobin level and adverse events in children with cancer presenting with fever in neutropenia. Plos one 2014; 9(7):e101696.

2. Maguire JL, Kulik DM, Laupacis A. Kuppermann N, Uleryk EM, Parkin PC. Clinical prediction rules for children: a systematic review. Pediatrics 2011; 128:e666-677.

3. Delebarre M, Marcher E, Mazingue F, Martinot A, Dubos F. Which decision rules meet methological standards in children with febrile neutropenia? Results of a systematic review and analysis. Pedia- tr Blood Cancer 2014; 61:1786-1791.

4. Alp S, Akova M. Management of febrile neutropenia in the era of bacterial resistance. Ther Adv Infect Dis 2013; 1: 37-43.

5. Bacteremia in hospitalized cancer patients with febrile neutropenia: a cohort study. Am J Infect Control 2014; 42: 74-6.

6. Madrid C, Díaz L, Combariza J, Gálvez K, Olaya V, Ramírez I, Donado J. Epidemiología de la neutropenia febril en adultos con neoplasia hematológica, en un período de 26 meses en el Hospital Pablo Tobón Uribe, Colombia. Rev Chilena Infectol 2013; 30:195-201. 
7. Freifeld AG, Bow EJ, Sepkowitz KA, Boeckh MJ, Ito JI, Mullen CA et al. Clinical practice guideline for the use of antimicrobial agents in neutropenic patients with cancer: 2010 update by the Infectious Diseases Society of America. Clin Infect Dis 2011; 52: e56-e92.

8. Abou Saleh M, Ngozi Mafiana R, Al Za'abi M, Vaishnav R, Al Kindi S, AlZakwani I. Epidemiology of chemotherapy-induced neutropenia at a tertiary university hospital in Oman. Int J Clin Pharm 2013; 35: 1036-9.

9. Bucaneve G, Micozzi A, Menichetti F, Martino P, Dionisi MS, Martinelli $\mathrm{G}$ et al. Levofloxacin to prevent bacterial infection in patients with cancer and neutropenia. N Engl J Med 2005; 353: 977987.

10. González-leal XJ, Molina-gamboa J, Bolaños-meade J, Villela L. Artículo original Aislamientos microbiológicos en pacientes con neutropenia febril. ¿Es apropiado el uso de las guías clínicas internacionales en México? Rev Hematol. 2013;14(81):113-9.

11. Ting J. Neutropenia febril. Tratado Med urgencias pediátricas. 2007;(Iccm):320-1.

12. Alp S, Akova M. Management of febrile neutropenia in the era of bacterial resistance. Ther Adv Infect Dis [Internet]. 2013 Feb [cited 2019 Mar 24];1(1):37-43. Available from: http://www.ncbi.nlm.nih. gov/pubmed/25165543
13. Freifeld AG, Bow EJ, Sepkowitz KA, Boeckh MJ, Ito JI, Mullen CA, et al. Clinical Practice Guideline for the Use of Antimicrobial Agents in Neutropenic Patients with Cancer: 2010 Update by the Infectious Diseases Society of America. 2011;52.

14. Trucchia S, Edith R. Universidad Nacional De Córdoba Facultad De Ciencias Médicas Modelo Predictivo De Bacteriemia En Pacientes. 2015, (1):34-41.

15. Daniel C, Silva B. Caracterizacion de la neutropenia febril en pacientes con leucemia linfoide aguda, tratados con quimioterapia de alto riesgo, atendidos en el instituto nacional de cancerologia desde 1 de enero al 31 de diciembre de 2008. 2009.

16. Hinojosa L, Carpio D Del. Bacteriemia asociada a neutropenia febril en pacientes hemato-oncológicos, su espectro bacteriano y patrón de susceptibilidad antibiótica. Rev Med Hered. 2014; 25:22-9.

17. Clínico H, Católica U, G RB, C IA, G GR, Garrido M, et al. Etiología de episodios de neutropenia febril en pacientes adultos con cáncer hematológico y de órganos sólidos en el Hospital Clínico Universidad Católica, Santiago-Chile. 2009;26(2):106-108. 